\title{
Model dependence of the neutrino-deuteron disintegration cross sections at low energies
}

\author{
B. Mosconi \\ Università di Firenze, Department of Physics, and \\ Istituto Nazionale di Fisica Nucleare, Sezione di Firenze, I-50019, Sesto Fiorentino (Firenze), Italy \\ P. Ricci \\ Istituto Nazionale di Fisica Nucleare, Sezione di Firenze, I-50019, Sesto Fiorentino (Firenze), Italy \\ E. Truhlík \\ Institute of Nuclear Physics ASCR, CZ-250 68 Řež, Czech Republic, and \\ Institute for Nuclear Theory and Department of Physics, University of Washington, Seattle, Washington 98195, USA \\ P. Vogel \\ Kellog Radiation Laboratory and Physics Department, Caltech, Pasadena, California 91125, and \\ Institute for Nuclear Theory and Department of Physics, University of Washington, Seattle, Washington 98195, USA
}

(Received 23 February 2007; published 25 April 2007)

\begin{abstract}
Model dependence of the reaction rates for the weak breakup of deuterons by low-energy neutrinos is studied starting from the cross sections derived from potential models and also from pionless effective field theory. Choosing the spread of the reaction yields, caused basically by the different ways the two-body currents are treated, as a measure of the model-dependent uncertainty, we conclude that the breakup reactions are $\sim 2-3 \%$ uncertain and that even the ratio of the charged to neutral current reaction rates is also $\sim 2 \%$ uncertain.
\end{abstract}

DOI: 10.1103/PhysRevC.75.044610

\section{INTRODUCTION}

The SNO collaboration [1-4], following the original suggestion by late Herb Chen [5], has convincingly shown that the flavor of solar neutrinos is not conserved. This was achieved by determining the yield of the deuteron disintegration in both neutral and charged current channels:

$$
\begin{aligned}
& v_{x}+d \rightarrow v_{x}^{\prime}+n+p, \\
& v_{e}+d \rightarrow e^{-}+p+p .
\end{aligned}
$$

The neutrino flux deduced from the neutral current reaction (1.1) agrees within errors with the standard solar model (SSM) [6], whereas the flux deduced from the charged current reaction (1.2) is smaller than the SSM prediction by a factor of $\sim 3$. The only reasonable way to interpret this result, and the other observations of solar neutrinos [7-12], is in terms of neutrino oscillations. This conclusion becomes inescapable when the reactor neutrino experiment KamLAND [13,14] is included in the corresponding fit.

To relate the yield of the reactions observed in SNO to the corresponding solar neutrino flux one needs to know the neutrino-deuteron breakup cross section. Consequently, the cross sections of the reactions (1.1) and (1.2) and the analogous ones initiated by antineutrinos,

$$
\begin{aligned}
& \bar{v}_{x}+d \rightarrow \bar{v}_{x}^{\prime}+n+p, \\
& \bar{v}_{e}+d \rightarrow e^{+}+n+n,
\end{aligned}
$$

have been carefully evaluated during the past two decades (see Refs. [15-20] and references therein). Here we wish to assess the uncertainties or model dependence involved in
PACS number(s): 25.30.Pt, 11.40.Ha, 25.10.+s, 26.65.+t

these evaluations related to the different ways the two-body exchange currents are treated.

The studies of the reactions (1.1)-(1.4) at low energies were performed in Refs. [15-18] based on the currents derived from elementary hadron amplitudes extracted in the tree approximation from the chiral Lagrangians and using nuclear wave functions generated from realistic nuclear potentials.

Alternatively, in Ref. [19], the cross sections derived in the next-to-next-to-leading order of the pionless effective field theory, were written in the form

$$
\sigma_{\mathrm{EFT}}\left(E_{v}\right)=a\left(E_{v}\right)+L_{1, A} b\left(E_{v}\right) .
$$

Tables of numerical values of the amplitudes $a\left(E_{v}\right)$ and $b\left(E_{v}\right)$ are given in Ref. [19] up to $20 \mathrm{MeV}$ in $1-\mathrm{MeV}$ steps.

In principle, the effective field theory provides a more fundamental approach to the study of nuclear phenomena, but it contains parameters that cannot be determined in reactions between elementary particles. The factor $L_{1, A}$ in Eq. (1.5) that parameterizes the effect of the isovector axial two-body current, is an example of such a constant. Its value can be determined from a measurement of any of the breakup processes (1.1)-(1.4). The analysis of various data [21,22] provides $L_{1, A}$ value, however, with a large error,

$$
L_{1, A}=3.6 \pm 5.5 \mathrm{fm}^{3} .
$$

Alternatively, the value of $L_{1, A}$ can be determined by comparing the cross sections (1.5) with the cross sections calculated employing the nuclear wave functions generated from realistic one-boson-exchange-potentials (OBEPs) and the one- and two-nucleon currents as it was done in the recent work [18]. The resulting values of $L_{1, A}$ were confined between 
the limits (see Table 2 in Ref. [18])

$$
4.4 \leqslant L_{1, A} \leqslant 7.2 \mathrm{fm}^{3} .
$$

To assess the global model dependence of the reaction rates for the breakup processes (1.1) and (1.2) we consider here the integral yield

$$
Y=\int_{0}^{\infty} \Phi_{8_{B}}\left(E_{v}\right) \sigma\left(E_{v}\right) d E_{v},
$$

where $\Phi_{8_{B}}\left(E_{v}\right)$ is the normalized spectrum corresponding to the decay of ${ }^{8} B$ [23] and the cross section $\sigma\left(E_{v}\right)$ is given as

$$
\sigma\left(E_{v}\right)=\int_{0}^{T_{l}^{\max }} \frac{d \sigma}{d T_{l}}\left(E_{v}, T_{l}\right) d T_{l}
$$

Here $T_{l}$ is the (kinetic) energy of the outgoing (charged) lepton. The information on the theoretical uncertainty or spread of $Y$ is obviously important for the detailed analysis of the data obtained from the SNO detector.

In Sec. II, we discuss briefly the methods and inputs necessary for the calculations and in Sec. III, we present the results. We conclude in Sec. IV. Further, in Appendix A, we present the reaction rates for the charged channel reaction (1.2) with the energy response function of the SNO detector taken into account, and in Appendix B, we collect the updated cross sections for all deuteron breakup reactions (1.1)-(1.4) up to (anti-)neutrino energies $E_{\nu}=20 \mathrm{MeV}$.

\section{METHODS AND INPUTS}

To obtain the cross sections one must first calculate the matrix elements of the weak nuclear currents (charged and neutral) between the initial and final nuclear states. Here we briefly describe the needed ingredients of these calculations. We follow the treatment described in detail in Section 4 of Ref. [18].

\section{A. Weak nuclear currents}

The weak nuclear current used to describe the neutral channel reaction (1.1) is

$$
j_{\mathrm{NC}, \mu}=\left(1-2 \sin ^{2} \theta_{W}\right) j_{\mu}^{3}-2 \sin ^{2} \theta_{W} j_{S \mu}+j_{5 \mu}^{3},
$$

where $\theta_{W}$ is the Weinberg angle, $j_{\mu}^{3}\left(j_{5 \mu}^{3}\right)$ is the third component of the weak vector (axial) current in the isospin space, and $j_{S \mu}$ is the isoscalar vector current. The weak hadron current, triggering the charged channel reaction (1.2), is

$$
j_{\mathrm{CC}, \mu}^{a}=j_{\mu}^{a}+j_{5 \mu}^{a}, \quad(a= \pm) .
$$

At low energies, the space component of the weak axial hadron current is the most important one.

The weak axial nuclear current $j_{5 \mu}^{a}$ for all three components, $a= \pm$ and 3 , consists of the one- and two-nucleon parts. There is practically no uncertainty associated with the one-body part. Hence we concentrate on the effects of the two-body currents. The weak axial nuclear two-body exchange current $j_{5 \mu}^{a}(2)$ that we consider here is of the OBE type with the $\pi-, \rho-, \omega-$, and $a_{1}$ exchanges. It can be divided [24] into the potential and nonpotential currents. The potential current of the range
$B, j_{5 \mu, B}^{a}(2$, pot), satisfies the nuclear partially conserved axial current (PCAC) equation,

$$
q_{\mu} j_{5 \mu, B}^{a}(2, \text { pot })=\left[\mathrm{V}_{\mathrm{B}}, \mathrm{j}_{50}^{\mathrm{a}}(1)\right]+\mathrm{if}_{\pi} \mathrm{m}_{\pi}^{2} \Delta_{\mathrm{F}}^{\pi}\left(\mathrm{q}^{2}\right) \mathcal{M}_{\mathrm{B}}^{\mathrm{a}}(2),
$$

where $V_{B}$ is the OBEP of the same range $B, j_{50}^{a}(1)$ is the one-body axial charge density and $\mathcal{M}_{B}^{a}(2)$ is the associated pion absorption/production exchange amplitude. Further $f_{\pi}$ is the pion decay constant, $m_{\pi}$ is the pion mass, and $\Delta_{F}^{\pi}$ is the pion propagator. This current is model independent and if a particular OBEP is used to generate the nuclear wave functions, then its effect can be calculated in a modelindependent way.

The main part of the nonpotential weak axial exchange current contains the model independed $\rho-\pi$ current and the $\Delta$ excitation currents that are model dependent. In our calculations, we shall adopt the $\pi-N-\Delta$ and $\rho-N-\Delta$ Lagrangians used for many years $[25,26]$ to study the $\pi N$ reactions and the pion photo- and electroproduction on a nucleon (model I) and also the gauge symmetric Lagrangians proposed recently $[27,28]$ (model II). The $\Delta$ excitation effect is in the model II suppressed due to the appearance of an additional factor $\left(M / M_{\Delta}\right)^{2} \approx 0.58$ $\left[M\left(M_{\Delta}\right)\right.$ is the nucleon ( $\Delta$ isobar) mass] in the exchange current operators.

Let us note that our model current II differs from an analogous current of Ref. [17]. That current is a purely phenomenological one, the potential part of which does not satisfy the PCAC constraint and the suppression of the $\Delta$ strength is achieved by reducing the $\Delta-N$ coupling to fit the Gamow-Teller matrix element in the triton beta decay.

\section{B. Nuclear potentials}

We use the Nijmegen I (NijmI), Nijmegen 93 (Nijm93) [29], and QG [30] one-boson-exchange potentials. The couplings and cutoffs, entering these potentials, are employed in our exchange currents. For comparison, we also consider the cross sections calculated from the AV18 potential, which is not an OBEP (see Table I of Ref. [16]).

\section{Extraction of $L_{1, A}$}

We extract the low energy constant $L_{1, A}$ from comparison of the cross sections based on the potential models and the EFT form $\sigma_{\mathrm{EFT}}$, see Eq. (1.5). For each of the $i$-th $1-\mathrm{MeV}$ bins we obtain the $L_{1, A}(i)$ value and take the corresponding average

$$
L_{1, A}=\frac{\sum_{i=1}^{N} L_{1, A}(i)}{N}, \quad L_{1, A}(i)=\frac{\sigma_{\mathrm{pot}, i}-a_{i}}{b_{i}},
$$

where $\sigma_{\mathrm{pot}, i}$ is the cross section, calculated in the potential model and for the $i$-th neutrino energy. We use $N=13$ for the reaction (1.1) and $N=14$ for the reaction (1.2), because for the solar neutrinos $E_{v} \leqslant 15 \mathrm{MeV}$ and $i=1$ correspond to the relevant reaction threshold. In addition, we extract $L_{1, A}$ also by the least-squares fit. It turns out that these two values of $L_{1, A}$ are not identical and provide somewhat different effective cross sections. We label the results for the reaction rates obtained with $L_{1, A}$ from Eq. (2.4) by $a v$, whereas the results calculated with $L_{1, A}$ from the least-squares fit are labeled lsf. 


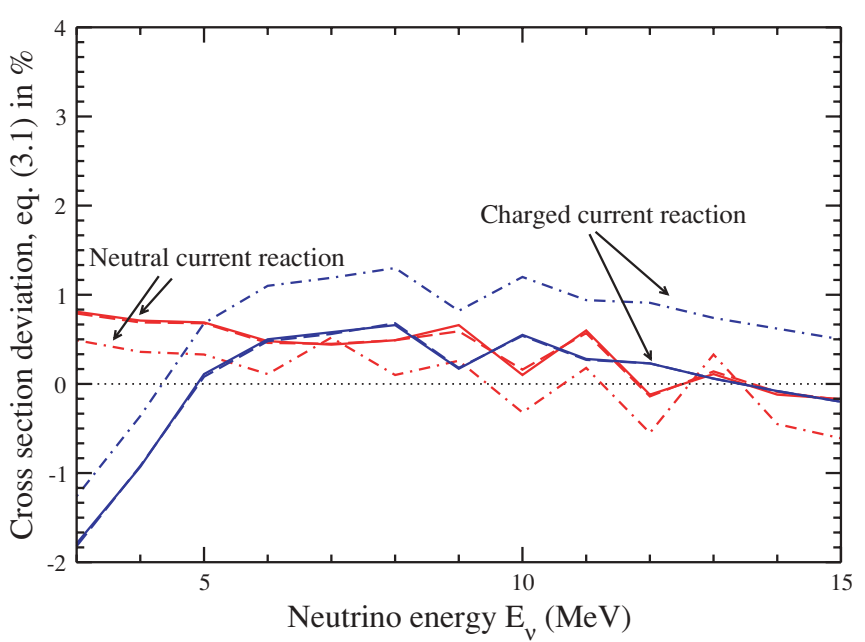

FIG. 1. (Color online) The differences $\delta_{i}^{a}$ in percentages [see Eq. (3.1)]. Full lines are based on model I and use $L_{1, A}^{\mathrm{lsf}}=5.2 \mathrm{fm}^{3}$ for ncd and $5.6 \mathrm{fm}^{3}$ for ccd; the dashed lines are for the same choice but for the model II, where $L_{1, A}^{\mathrm{lsf}}=3.8 \mathrm{fm}^{3}$ for ncd and $4.2 \mathrm{fm}^{3}$ for ccd. Finally, the dot-dashed lines are for the model I with $L_{1, A}^{\mathrm{av}}=5.5 \mathrm{fm}^{3}$ for ncd and $5.1 \mathrm{fm}^{3}$ for ccd.

\section{RESULTS AND DISCUSSION}

To be compatible with the calculations [19], we use the same weak interaction constants, $G_{F}=1.166 \times 10^{-5} \mathrm{GeV}^{-2}$, $g_{A}=-1.26, \cos \theta_{C}=0.975$, which differ only slightly from the constants employed in Ref. [16]. In Ref. [18] the contributions from the multipole $J=1$ were calculated for the transition $d \rightarrow{ }^{1} S_{0}$ both for the one- and two-nucleon currents and also for the transitions $d \rightarrow{ }^{3} P_{j_{f}}, j_{f}=0,1,2$ for the onenucleon current. Here the computation code already contains the contributions from all multipoles $J=0,1,2,3$ and the transitions $d \rightarrow{ }^{2 S+1} L_{j_{f}}, j_{f}=0,1,2$ for the one-nucleon current.

\section{A. Energy dependence}

The extracted values of the low-energy constant $L_{1, A}$ depend on the way it was determined (averaging or least squares) and on the potential used. It varied in the limits $3.8 \leqslant L_{1, A} \leqslant 5.7 \mathrm{fm}^{3}$ for the neutral current reaction (ncd) (1.1) and $3.9 \leqslant L_{1, A} \leqslant 6.4 \mathrm{fm}^{3}$ for the charged current reaction (ccd) (1.2). Alternatively, $L_{1, A}$ can be determined by requiring specifically for the problem of solar ${ }^{8} \mathrm{~B}$ neutrinos that the yields $Y$, Eq. (1.8), are identical whether one uses the corresponding potential model cross section or the EFT one. The ranges of the $L_{1, A}$ values are then quite similar to those shown above, namely $4.2 \leqslant L_{1, A} \leqslant 5.6 \mathrm{fm}^{3}$ for ncd and $4.4 \leqslant L_{1, A} \leqslant 6.7 \mathrm{fm}^{3}$ for ccd.

In fact, the energy-dependent parameter $L_{1, A}(i)$ is not really a constant (see also Tables 3 and 4 of Ref. [18]). Instead its values varied, even for a fixed choice of the potential and method of $L_{1 A}$ extraction. In other words, this means that the cross sections evaluated with a single $L_{1 A}$, obtained either by averaging (av) or by the least-squares fit (lsf) as described above and using Eq. (1.5) differ from the cross sections based on the potential model in an energy-dependent way. We illustrate the energy dependence of such differences in Fig. 1, where we plot $\delta_{i}^{a}$ defined as

$$
\delta_{i}^{a}=1-\frac{\sigma_{\mathrm{EFT}}\left[L_{1, A}^{a}(i)\right]}{\sigma_{\mathrm{pot}, i}}
$$

for both methods of $L_{1, A}$ extraction ( $a=1 \mathrm{sf}$, av). For the potential model, we chose the Nijmegen I potential. Other potential models used in this work provide similar picture.

As seen in Fig. 1 the neutral current cross section behaves in a regular smooth way, and the EFT and potential model based methods give cross sections that differ by not more than $1 \%$ over the relevant energy range. The cross section for the charged current reaction exhibits somewhat stronger variations with energy, in particular for the lowest energy bins. The origin of this effect remains unknown so far.

The deviations illustrated in Fig. 1 cause a corresponding variations with energy of the partial values $L_{1, A}(i)$. One can quantify this by pointing out that for the values corresponding to the full lines in Fig. 1 the mean-square deviations

$$
\delta L_{1, A}=\left(\left\langle L_{1, A}^{2}\right\rangle-\left\langle L_{1, A}\right\rangle^{2}\right)^{1 / 2}
$$

TABLE I. Reaction rates $Y\left(\times 10^{-42} \mathrm{~cm}^{2}\right)$ for the weak deuteron breakup by the ${ }^{8} \mathrm{~B}$ neutrinos in the charged (ccd) and neutral current channels (ncd) using the model currents I and II. The yield ratio is $R_{i}=Y_{i}(\mathrm{ccd}) / Y_{i}(\mathrm{ncd})$, for $i=\mathrm{I}$, II. In model II (see Sec. II A), the $\Delta$ excitation currents are suppressed by a factor of $\approx 0.58$. In the columns labeled by NijmI, Nijm93, and QG, the cross sections are calculated with the wave functions generated from these potentials (see Sec. II B), the cross sections of the column AV18 are taken from Table I of Ref. [16]. The cross sections of the columns labeled by lsf and av, respectively, are obtained from Eq. (1.5) with the constant $L_{1, A}$ calculated by the least square fit [using Eq. (2.4)]. In the last column $\Delta S / S$ is the maximum deviation of the quantity corresponding to the given row.

\begin{tabular}{lllllllllllllll}
\hline \hline & & NijmI & lsf & av & Nijm93 & lsf & av & AV18 & lsf & av & QG & lsf & av & $\Delta S / S(\%)$ \\
\hline$Y_{\mathrm{I}}$ & ccd & 1.205 & 1.200 & 1.193 & 1.217 & 1.213 & 1.205 & 1.210 & 1.209 & 1.207 & - & - & - \\
$Y_{\mathrm{I}}$ & ncd & 0.470 & 0.468 & 0.470 & 0.471 & 0.469 & 0.471 & 0.470 & 0.470 & 0.470 & 0.470 & 0.468 & 0.470 \\
$R_{\mathrm{I}}$ & & 2.56 & 2.58 & 2.54 & 2.58 & 2.59 & 2.56 & 2.57 & 2.57 & 2.57 & - & - & - & 0.6 \\
$Y_{\mathrm{II}}$ & ccd & 1.185 & 1.181 & 1.173 & 1.195 & 1.191 & 1.183 & - & - & - & - & - & - \\
$Y_{\mathrm{II}}$ & ncd & 0.462 & 0.460 & 0.462 & 0.462 & 0.460 & 0.462 & - & - & - & 0.462 & 0.460 & 0.460 \\
$R_{\mathrm{II}}$ & & 2.57 & 2.57 & 2.54 & 2.59 & 2.59 & 2.56 & - & - & - & - & - & - & 0.4 \\
\hline \hline
\end{tabular}


TABLE II. Reaction rates calculated with the one-nucleon currents only. For the notations see Table I.

\begin{tabular}{lcccccccccc}
\hline \hline & NijmI & lsf & av & Nijm93 & lsf & av & QG & lsf & av & $\Delta S / S(\%)$ \\
\hline$Y_{\text {cdd }}^{\text {IA }}$ & 1.150 & 1.146 & 1.138 & 1.150 & 1.146 & 1.138 & - & - & - & 1.0 \\
$Y_{\text {ncd }}^{\text {IA }}$ & 0.447 & 0.446 & 0.447 & 0.445 & 0.444 & 0.445 & 0.449 & 0.446 & 0.449 & 1.1 \\
$R$ & 2.57 & 2.57 & 2.55 & 2.58 & 2.58 & 2.56 & - & - & - & 1.0 \\
\hline \hline
\end{tabular}

are $\delta L_{1, A}=0.4$, i.e., much smaller than $L_{1, A}^{\mathrm{av}}=5.5$ for ncd, whereas for ccd $\delta L_{1, A}=1.8$ that is a bit larger but still considerably smaller than the $L_{1, A}^{\mathrm{av}}=5.1$.

\section{B. Global features}

We characterize the global rates by the corresponding reaction yields (1.8). The differences between these yields is a measure of the theoretical uncertainty of the corresponding cross sections. The results of the calculations for the reactions (1.1) and (1.2) are presented in Table I.

It is seen from Table I that, first of all, despite the slight energy dependence of the ccd cross section discussed above, the EFT reaction yields agree reasonably well with the corresponding quantities based on the potential models. However, when $L_{1, A}^{\mathrm{av}}$ is used for the ccd channel, the corresponding yield appears to be systematically smaller. It turns out that the yield ratios $R_{i}$ do not depend sensitively on the current model but depend somewhat more on the choice of the nuclear force and on the method of the extraction of $L_{1, A}$. In contrast, the reaction yields depend more on the choice of the current model and less on the choice of the potential.

As a measure of the uncertainty we shall use the largest relative difference of the corresponding yield. Using such criterion, an uncertainty of $\approx 2 \%$ in the calculations of the ratio of the reaction rates follows, whereas the uncertainty of the reaction rate is $\approx 2.3 \%(3.3 \%)$ in the neutral (charged) channel stemming mostly from the difference between the models I and II. Let us note that the radiative corrections will enhance the reaction rates by $\approx 1.5 \%(2 \%)$ in the neutral (charged) channel [31].

In Table II analogous results obtained when only the onenucleon currents are included are presented. It follows from comparison of Tables I and Table II that the effect of the meson exchange currents, $\delta_{\mathrm{MEC}}^{i}$, depends on the potential model and varies as $4.6 \% \leqslant \delta_{\mathrm{MEC}}^{I} \leqslant 5.7 \%$ and $2.4 \% \leqslant \delta_{\mathrm{MEC}}^{I I} \leqslant 3.8 \%$ for the model currents I and II, respectively.
The reaction rates obtained with the one-nucleon currents only still provide nonvanishing values of $L_{1, A}$ because the strong interaction part of the problem is treated in the potential model and in the EFT differently. The corresponding values of the effective parameter $L_{1, A}$ vary in the limits

$$
1.1 \leqslant L_{1, A} \leqslant 2.0 \mathrm{fm}^{3}
$$

If one takes in Eq. (1.5) $L_{1, A}=0$, then

$$
Y_{\mathrm{ccd}}^{\mathrm{IA}, \mathrm{EFT}}=1.120 \times 10^{-42} \mathrm{~cm}^{2},
$$

for the charged channel reaction, and

$$
Y_{\mathrm{ncd}}^{\mathrm{IA}, \mathrm{EFT}}=0.437 \times 10^{-42} \mathrm{~cm}^{2},
$$

for the neutral channel reaction. These values are smaller by $2-3 \%$ than the reaction rates of Table II. However, the rate ratio $R$ is essentially independent of the two-body current.

In the charged channel reaction (1.2) the electron spectrum is also measured in SNO. The number of events with the observed electron kinetic energy $T$ depends then on the response of the detector function. The ccd reaction yield is then (see Ref. [4])

$$
\begin{aligned}
Y_{R}= & \int_{0}^{\infty} \int_{0}^{T_{e}^{\max }} \int_{T_{\mathrm{th}}}^{\infty} \Phi_{8_{B}}\left(E_{v}\right) \frac{d \sigma}{d T_{e}}\left(E_{v}, T_{e}\right) \\
& \times R\left(T_{e}, T\right) d E_{v} d T_{e} d T \\
\equiv & \int_{0}^{\infty} \Phi_{8_{B}}\left(E_{v}\right) \sigma_{R}\left(E_{v}\right) d E_{\nu}
\end{aligned}
$$

where $T_{e}$ is the true recoil electron kinetic energy and $R\left(T_{e}, T\right)$ is the energy response function,

$$
R\left(T_{e}, T\right)=\frac{1}{\sqrt{2} \pi \sigma_{T}} \exp \left[-\frac{\left(T_{e}-T\right)^{2}}{2 \sigma_{T}^{2}}\right]
$$

\begin{tabular}{|c|c|c|c|c|c|c|c|c|c|c|c|c|c|}
\hline$E_{v}(\mathrm{MeV})$ & 3 & 4 & 5 & 6 & 7 & 8 & 9 & 10 & 11 & 12 & 13 & 14 & 15 \\
\hline$\sigma$ & 0.0456 & 0.1536 & 0.3406 & 0.6144 & 0.9812 & 1.444 & 2.008 & 2.673 & 3.444 & 4.322 & 5.310 & 6.410 & 7.622 \\
\hline$\sigma_{R}$ & $7(-7)$ & $1(-4)$ & 0.0051 & 0.0708 & 0.3781 & 0.9999 & 1.746 & 2.521 & 3.354 & 4.265 & 5.279 & 6.396 & 7.623 \\
\hline$\sigma_{R}^{s}(5.0)$ & $1(-6)$ & $2(-4)$ & 0.0059 & 0.0749 & 0.3823 & 0.9984 & 1.743 & 2.518 & 3.352 & 4.263 & 5.277 & 6.393 & 7.619 \\
\hline$\sigma_{R}^{s}(5.5)$ & $4(-7)$ & $5(-5)$ & 0.0023 & 0.0358 & 0.2361 & 0.7744 & 1.564 & 2.412 & 3.284 & 4.222 & 5.246 & 6.370 & 7.597 \\
\hline
\end{tabular}

TABLE III. The neutrino energy-dependent cross sections (in $10^{-42} \mathrm{~cm}^{2}$ ) for the ccd reaction (1.2) calculated using the NijmI potential and the model I currents. The cross section $\sigma$ is given in Eq. (1.9); $\sigma_{R}$-overlap integral of the cross section with the response function as defined in the second line of Eq. (3.6), pure heavy water phase; $\sigma_{R}^{s}(5.0)$-salt phase with $T_{\text {th }}=5.0 \mathrm{MeV} ; \sigma_{R}^{s}(5.5)$-salt phase with $T_{\text {th }}=5.5 \mathrm{MeV}$. The shorthand $a(-n)$ means $a \times 10^{-n}$. 
For the pure heavy water phase the resolution width $\sigma_{T}$ was taken in the form,

$$
\begin{aligned}
\sigma_{T}(T) & =-0.0648+0.331 \sqrt{T}+0.0425 T, \\
T_{\mathrm{th}} & =5.0 \mathrm{MeV},
\end{aligned}
$$

whereas for the salt phase [2] it was,

$$
\begin{aligned}
\sigma_{T}(T) & =-0.145+0.392 \sqrt{T}+0.0353 T, \\
T_{\text {th }} & =5.5 \mathrm{MeV},
\end{aligned}
$$

To see the effect of the response function and threshold we compare in Table III the cross section without the response and the effective cross section $\sigma_{R}\left(E_{v}\right)$ of Eq. (3.6). To emphasize the crucial role of the threshold we include a line corresponding to a hypothetical lower threshold of $5 \mathrm{MeV}$ for the salt phase.

Despite the very important effects of the thresholds and response function we believe that the global characteristics used in Table I can be used as a measure of the theoretical uncertainty associated with the relative spread of the cross sections caused be the different model assumptions.

\section{CONCLUSIONS}

We have evaluated the spread of the calculated cross sections, and of the corresponding reaction yields, for the electron neutrino from ${ }^{8} \mathrm{~B}$ decay induced deuteron breakup reactions. The spread is caused by the different choices of the one-boson-exchange potentials, and in particular, by the ways the $\Delta$ excitation currents are treated. Choosing such spread as a measure of the uncertainty we conclude that the neutral current breakup is $\sim 2.3 \%$ uncertain, and the charged current one is $\sim 3.3 \%$ uncertain. The ratio of the charged to neutral current reaction rates is then $\sim 2 \%$ uncertain, using this criterion. These uncertainties are smaller, but basically comparable, to the full effect of the two-body currents. Thus, we have to conclude that the evaluation of the effect of the two-body currents remains to be quite uncertain. We have verified that our conclusions are not changed noticeably when the realistic thresholds and resolution functions of the SNO experiment are used.

\section{ACKNOWLEDGMENTS}

This work was supported by the grant GAČR 202/06/0746 and by Ministero dell' Istruzione, dell' Università e della Ricerca of Italy (PRIN 2006). Two of us (E.T. and P.V.) thank the INT at the University of Washington for the hospitality and the Department of Energy for partial support during the initial stage of this work. We thank J. Formaggio for very useful discussions.
TABLE IV. The values of the reaction rates $Y_{R}\left(\times 10^{-42} \mathrm{~cm}^{2}\right)$ for the charged current reaction (1.2) calculated according to Eq. (3.6) and using the potential models NijmI and Nijm93 and the current models I and II. The values of $Y_{R}^{s}$ and $Y_{R, I A}^{s}$ are calculated with the resolution function (3.9), for comparison the values of $Y_{R, I A}^{s}$ and $Y_{R, \mathrm{IA}}$ are obtained with the one-nucleon currents only. The ratios $R$ are always obtained by using the total yield from the column at the left and the related NC total yield either from Table I or Table II.

\begin{tabular}{lcccccc}
\hline \hline & $Y_{R, \mathrm{I}}^{s}$ & $R_{R, \mathrm{I}}^{s}$ & $Y_{R, \mathrm{II}}^{s}$ & $R_{R, \mathrm{II}}^{s}$ & $Y_{R, I A}^{s}$ & $R_{R, I A}^{s}$ \\
\hline NijmI & 0.816 & 1.74 & 0.803 & 1.74 & 0.781 & 1.75 \\
Nijm93 & 0.824 & 1.75 & 0.810 & 1.75 & 0.781 & 1.76 \\
& $Y_{R, \mathrm{I}}$ & $R_{R, \mathrm{I}}$ & $Y_{R, \mathrm{II}}$ & $R_{R, \mathrm{II}}$ & $Y_{R, I A}$ & $R_{R, I A}$ \\
NijmI & 0.898 & 1.91 & 0.884 & 1.91 & 0.859 & 1.92 \\
Nijm93 & 0.907 & 1.93 & 0.891 & 1.93 & 0.859 & 1.93 \\
\hline \hline
\end{tabular}

\section{APPENDIX A: CHARGED CHANNEL REACTION RATES, INCLUDING THE RESOLUTION FUNCTION AND THRESHOLDS OF THE SNO DETECTOR}

Here we repeat some of the previous calculations, but take into account the resolution function and threshold of the SNO detector. The reaction rates for the charged channel reaction (1.2) are presented in Table IV.

It is seen from Table IV that the effect of the meson exchange currents is $4.3 \% \leqslant \delta_{\mathrm{MEC}}^{I} \leqslant 6.0 \%$ and $2.7 \% \leqslant$ $\delta_{\text {MEC }}^{I I} \leqslant 4.0 \%$ for the model currents $I$ and $I I$, respectively, and it follows closely the effect obtained above without taking into account the response function of the detector, though shifted by $\approx 0.3 \%$ upward.

The ratio $R=Y_{\mathrm{CC}} / Y_{\mathrm{NC}}$ was calculated earlier by Bahcall and Lisi [32], who obtained

$$
R=1.882 \pm 0.042
$$

using the response function (3.7) with the resolution

$$
\sigma_{T}(T)=1.1 \sqrt{0} .1 T, \quad T_{\mathrm{th}}=5.0 \mathrm{MeV} .
$$

Adopting such a response function, we obtained for the NijmI wave functions $Y_{R, I}\left(R_{R, I}\right)=0.889(1.89)$ and $Y_{R, I}^{s}\left(R_{R, I}^{s}\right)=$ $0.806(1.71)$, for the pure heavy water and salt phases, respectively. The result for $R_{R, I}=1.89$ is in a very good agreement with Eq. (A1).

Comparison with Table I shows that the reaction yields $Y_{R}^{s}$ $\left(Y_{R}\right)$ are reduced by the factor $\approx 0.68(0.75)$, presumably due to the presence of the threshold $T_{\text {th }}$. If one takes $T_{\text {th }}=5.0 \mathrm{MeV}$ for the salt phase, one obtains for $Y_{R, i}^{s}$ values that coincide with $Y_{R, i}$ of Table IV within three digits. This is so, because the cross sections $\sigma_{R}$ and $\sigma_{R}^{s}(5.0)$ are close to each other for $E_{v} \geqslant 7 \mathrm{MeV}$ (see Table III).

\section{APPENDIX B: COMPARISON OF THE CROSS SECTIONS}

Here we compare our updated cross sections with the cross sections of Refs. [16] and [19] up to (anti-)neutrino energies $E_{v}=20 \mathrm{MeV}$. Tables V and VI supersede Tables 3 and 4 of Ref. [18] and Tables 3-6 of Ref. [33]. 
TABLE V. Cross sections and the differences, in percentages, between the cross sections for the reactions (1.1) and (1.3). In the first column, $E_{v}[\mathrm{MeV}]$ is the neutrino energy, in the second column, $\sigma_{\mathrm{NijmI}}$ (in $10^{-42} \times \mathrm{cm}^{2}$ ) is the cross section, calculated with the NijmI nuclear wave functions, $g_{A}=-1.26$ and $G_{F}=1.166 \times 10^{-5} \mathrm{GeV}^{-2}$, i.e., the weak interaction parameters used in Ref. [19]. In column 3 is the difference between $\sigma_{\mathrm{NijmI}}(\mathrm{I})$ and the EFT cross section (1.5) $\sigma_{\mathrm{EFT}}$, calculated with the corresponding constant $L_{1, A}^{\mathrm{av}}$ given in the parentheses. The difference between $\sigma_{\mathrm{NSGK}}$ taken from Table I of Ref. [16] and $\sigma_{\mathrm{EFT}}$ is given in column $4(\mathrm{~N})$. Further, $\Delta_{1(2)}$ is the difference between the cross sections $\sigma_{\mathrm{NijmI}}\left(\sigma_{\mathrm{Nijm} 93}\right)$ and $\sigma_{\mathrm{NSGK}}$. In this case, our cross sections are calculated with $g_{A}=-1.254$ [16]. The second part of the table is an analog for the reaction (1.3).

\begin{tabular}{|c|c|c|c|c|c|c|c|c|c|c|c|}
\hline \multicolumn{6}{|c|}{$v_{x}+d \rightarrow v_{x}^{\prime}+n p$} & \multicolumn{6}{|c|}{$\bar{v}_{x}+d \rightarrow \bar{v}_{x}^{\prime}+n p$} \\
\hline$E_{v}$ & $\sigma_{\mathrm{NijmI}}$ & $I(5.3)$ & $N(5.4)$ & $\Delta_{1}$ & $\Delta_{2}$ & $E_{\bar{v}}$ & $\sigma_{\mathrm{NijmI}}$ & $I(5.6)$ & $N(5.5)$ & $\Delta_{1}$ & $\Delta_{2}$ \\
\hline 3 & 0.00335 & 0.6 & 0.4 & -0.9 & -0.5 & 3 & 0.00332 & 0.0 & 0.1 & -0.9 & -0.4 \\
\hline 4 & 0.0307 & 0.6 & 0.2 & -0.6 & 0.3 & 4 & 0.0302 & 0.5 & 0.2 & -0.5 & 0.4 \\
\hline 5 & 0.0949 & 0.5 & 0.2 & -0.8 & -0.4 & 5 & 0.0930 & 0.3 & 0.1 & -0.7 & -0.3 \\
\hline 6 & 0.201 & 0.3 & 0.1 & -0.8 & -0.9 & 6 & 0.196 & 0.5 & 0.3 & -0.7 & -0.8 \\
\hline 7 & 0.353 & 0.3 & 0.1 & -0.9 & -1.0 & 7 & 0.343 & 0.1 & 0.1 & -0.8 & -0.9 \\
\hline 8 & 0.553 & 0.3 & 0.2 & -0.9 & -0.6 & 8 & 0.533 & 0.9 & 0.8 & -0.7 & -0.3 \\
\hline 9 & 0.801 & 0.5 & 0.4 & -1.0 & -0.8 & 9 & 0.768 & 0.3 & 0.2 & -0.8 & -0.5 \\
\hline 10 & 1.099 & -0.1 & -0.1 & -1.0 & -0.9 & 10 & 1.049 & 0.2 & 0.2 & -0.8 & -0.7 \\
\hline 11 & 1.447 & 0.4 & 0.5 & -1.1 & -1.0 & 11 & 1.373 & -0.2 & -0.2 & -0.9 & -0.8 \\
\hline 12 & 1.848 & -0.3 & -0.3 & -1.1 & -0.7 & 12 & 1.744 & -0.3 & -0.4 & -0.8 & -0.4 \\
\hline 13 & 2.299 & -0.1 & 0.0 & -1.2 & -0.9 & 13 & 2.158 & -0.3 & -0.2 & -0.9 & -0.6 \\
\hline 14 & 2.802 & -0.2 & 0.0 & -1.3 & -1.0 & 14 & 2.616 & -0.2 & -0.2 & -0.9 & -0.6 \\
\hline 15 & 3.359 & -0.3 & -0.1 & -1.3 & -1.1 & 15 & 3.118 & -0.3 & -0.2 & -1.0 & -0.7 \\
\hline 16 & 3.968 & -0.5 & -0.3 & -1.4 & -1.2 & 16 & 3.663 & -0.2 & -0.1 & -1.0 & -0.8 \\
\hline 17 & 4.631 & -0.7 & -0.4 & -1.4 & -1.3 & 17 & 4.252 & -0.4 & -0.2 & -1.0 & -0.9 \\
\hline 18 & 5.348 & -0.6 & -0.3 & -1.4 & -1.6 & 18 & 4.882 & -0.4 & -0.3 & -1.1 & -1.2 \\
\hline 19 & 6.119 & -0.7 & -0.4 & -1.5 & -1.6 & 19 & 5.555 & -0.5 & -0.3 & -1.1 & -1.3 \\
\hline 20 & 6.949 & -0.9 & -0.6 & -1.5 & -1.7 & 20 & 6.273 & -0.5 & -0.2 & -1.2 & -1.3 \\
\hline
\end{tabular}

TABLE VI. Cross sections and the differences, in percentages, between the cross sections for the reactions (1.2) and (1.4). For the notation, see Table V. In addition, $\cos \theta_{C}=0.975$ is used for comparison with the EFT, whereas $\cos \theta_{C}=0.9749$ when we compare our cross sections with Ref. [16].

\begin{tabular}{|c|c|c|c|c|c|c|c|c|c|c|c|}
\hline \multicolumn{6}{|c|}{$v_{e}+d \rightarrow e^{-}+p p$} & \multicolumn{6}{|c|}{$\bar{v}_{e}+d \rightarrow e^{+}+n n$} \\
\hline$E_{v}$ & $\sigma_{\mathrm{NijmI}}$ & $I(5.1)$ & $N(6.0)$ & $\Delta_{1}$ & $\Delta_{2}$ & $E_{\bar{v}}$ & $\sigma_{\mathrm{NijmI}}$ & $I(5.2)$ & $N(5.6)$ & $\Delta_{1}$ & $\Delta_{2}$ \\
\hline 2 & 0.00341 & -5.5 & -0.7 & -6.7 & -5.9 & 2 & - & - & - & - & - \\
\hline 3 & 0.0456 & -1.2 & -0.4 & -2.7 & -1.9 & 3 & - & - & - & - & - \\
\hline 4 & 0.154 & -0.4 & -0.6 & -1.7 & -0.8 & 4 & - & - & - & - & - \\
\hline 5 & 0.341 & 0.6 & 0.1 & -1.4 & -0.5 & 5 & 0.0274 & -2.0 & -1.0 & -2.3 & -1.5 \\
\hline 6 & 0.614 & 1.0 & 0.3 & -1.3 & -0.4 & 6 & 0.117 & -0.6 & -0.1 & -1.9 & -1.1 \\
\hline 7 & 0.981 & 1.1 & 0.4 & -1.3 & -0.3 & 7 & 0.278 & -0.4 & -0.2 & -1.6 & -0.7 \\
\hline 8 & 1.444 & 1.2 & 0.5 & -1.3 & -0.3 & 8 & 0.515 & -0.1 & -0.1 & -1.4 & -0.4 \\
\hline 9 & 2.008 & 0.7 & 0.0 & -1.4 & -0.3 & 9 & 0.832 & -0.1 & -0.2 & -1.3 & -0.3 \\
\hline 10 & 2.673 & 1.1 & 0.5 & -1.4 & -0.4 & 10 & 1.230 & 0.5 & 0.3 & -1.3 & -0.2 \\
\hline 11 & 3.444 & 0.8 & 0.3 & -1.5 & -0.5 & 11 & 1.708 & 0.4 & 0.2 & -1.3 & -0.2 \\
\hline 12 & 4.322 & 0.9 & 0.3 & -1.6 & -0.5 & 12 & 2.265 & 0.4 & 0.1 & -1.2 & -0.1 \\
\hline 13 & 5.310 & 0.7 & 0.2 & -1.6 & -0.6 & 13 & 2.903 & 0.3 & 0.0 & -1.2 & -0.1 \\
\hline 14 & 6.410 & 0.6 & 0.2 & -1.7 & -0.6 & 14 & 3.618 & 0.5 & 0.2 & -1.2 & -0.1 \\
\hline 15 & 7.622 & 0.5 & 0.1 & -1.7 & -0.6 & 15 & 4.411 & 0.3 & 0.0 & -1.3 & -0.1 \\
\hline 16 & 8.936 & 0.1 & -0.1 & -1.9 & -0.8 & 16 & 5.280 & 0.3 & 0.1 & -1.3 & -0.1 \\
\hline 17 & 10.37 & 0.0 & -0.2 & -2.1 & -1.0 & 17 & 6.225 & 0.4 & 0.2 & -1.3 & -0.2 \\
\hline 18 & 11.93 & -0.1 & -0.1 & -2.1 & -1.1 & 18 & 7.244 & 0.5 & 0.4 & -1.4 & -0.2 \\
\hline 19 & 13.61 & -0.1 & -0.1 & -2.2 & -1.1 & 19 & 8.335 & 0.3 & 0.2 & -1.4 & -0.2 \\
\hline 20 & 15.42 & -0.2 & -0.3 & -2.2 & -1.1 & 20 & 9.498 & 0.4 & 0.3 & -1.5 & -0.3 \\
\hline
\end{tabular}


[1] Q. R. Ahmad et al., Phys. Rev. Lett. 87, 071301 (2001); Phys. Rev. Lett. 89, 011301 (2002); Phys. Rev. Lett. 89, 011302 (2002).

[2] S. N. Ahmed et al., Phys. Rev. Lett. 92, 181301 (2004).

[3] B. Aharmim et al., Phys. Rev. C 72, 055502 (2005).

[4] B. Aharmim et al., nucl-ex/0610020.

[5] H. H. Chen, Phys. Rev. Lett. 55, 1534 (1985).

[6] J. N. Bahcall and M. Pinsonneault, Rev. Mod. Phys. 67, 781 (1995).

[7] B. T. Cleveland et al., Astrophys. J. 496, 505 (1998).

[8] J. Hosaka et al., Phys. Rev. D 73, 112001 (2006).

[9] Y. Fukuda et al., Phys. Rev. Lett. 77, 1683 (1996).

[10] J. N. Abdurashitov et al., Phys. Rev. C 60, 055801 (1999).

[11] W. Hampel et al., Phys. Lett. B447, 127 (1999).

[12] M. Altmann et al., Phys. Lett. B616, 174 (2005).

[13] T. Araki et al., Phys. Rev. Lett. 90, 021802 (2003).

[14] T. Araki et al., Phys. Rev. Lett. 94, 081801 (2005).

[15] S. Ying, W. C. Haxton, and E. M. Henley, Phys. Rev. C 45, 1982 (1992).

[16] S. Nakamura, T. Sato, V. Gudkov, and K. Kubodera, Phys. Rev. C 63, 034617 (2001).

[17] S. Nakamura, T. Sato, S. Ando, T.-S. Park, F. Myhrer, V. Gudkov, and K. Kubodera, Nucl. Phys. A707, 561 (2002).

[18] B. Mosconi, P. Ricci, and E. Truhlík, Nucl. Phys. A772, 81 (2006).

[19] M. Butler, J.-W. Chen, and X. Kong, Phys. Rev. C 63, 035501 (2001).
[20] S. Ando, Y. H. Song, T.-S. Park, H. W. Fearing, and K. Kubodera, Phys. Lett. B555, 49 (2003).

[21] A. B. Balantekin and H. Yüksel, Int. J. Mod. Phys. E 14, 39 (2005).

[22] M. Butler, J.-W. Chen, and P. Vogel, Phys. Lett. B549, 26 (2002).

[23] J. N. Bahcall and B. R. Holstein, Phys. Rev. C 33, 2121 (1986).

[24] B. Mosconi, P. Ricci, and E. Truhlík, Eur. Phys. J. A 25, 283 (2005).

[25] M. G. Olsson and E. T. Osypowski, Nucl. Phys. B87, 399 (1975).

[26] R. M. Davidson, N. C. Mukhopadhyay, and R. S. Wittman, Phys. Rev. D 43, 71 (1991).

[27] V. Pascalutsa and R. Timmermans, Phys. Rev. C 60, 042201(R) (1999).

[28] V. Pascalutsa, Phys. Lett. B503, 85 (2001).

[29] V. G. J. Stoks, R. A. M. Klomp, C. P. F. Terheggen, and J. J. de Swart, Phys. Rev. C 49, 2950 (1994).

[30] P. Obersteiner, W. Plessas, and E. Truhlík, in Proceedings of the XIII International Conference on Particles and Nuclei, Perugia, Italy, June 28-July 2, 1993, edited by A. Pascolini (World Scientific, Singapore, 1994), p. 430.

[31] A. Kurylov, M. J. Ramsey-Musolf, and P. Vogel, Phys. Rev. C 65, 055501 (2002).

[32] J. N. Bahcall and E. Lisi, Phys. Rev. D 54, 5417 (1996).

[33] B. Mosconi, P. Ricci, and E. Truhlík, Eur. Phys. J. A 27, 67 (2006). 